\title{
Evaluation of Ab Externo Subretinal Bands Removal During Pars Plana Vitrectomy for Rhegmatogenous Retinal Detachment Complicated by Proliferative Vitreoretinopathy
}

\author{
Samir Elbaha \\ Alexandria University \\ Mohammed Ghoneem \\ Alexandria University \\ Amir Abousamra \\ Alexandria University \\ Mahmoud Abouhussein ( $\sim$ nad03alaa@yahoo.com ) \\ Alexandria University
}

\section{Research Article}

Keywords: Rhegmatogenous retinal detachment, Parsplana vitrectomy, subretinal bands

Posted Date: January 31st, 2022

DOI: https://doi.org/10.21203/rs.3.rs-1258625/v1

License: (c) (1) This work is licensed under a Creative Commons Attribution 4.0 International License.

Read Full License 


\section{Abstract \\ Background}

To compare the safety and efficacy of Ab-externo subretinal bands removal in comparison with classical Ab-interno approach during pars plana vitrectomy for primary rhegmatogenous retinal detachment.

\section{Methods}

Subjects aged 28-62 years with primary RRD complicated by proliferative vitreoretinopathy (PVR) with subretinal bands interfering with retinal flattening were treated by pars plana vitrectomy (PPV) and silicone oil injection. Subretinal bands were removed using the classical $A B$ interno approach through one or more retinotomies in ten patients (group $A$ ) and using $A B$ externo approach in twenty cases (group B). Post-operative follow-up visits occurred at 1 day, 1 week, 1 month, and 3 months, after surgery. The main outcomes were assessment of subretinal bands removal efficacy, documentation of complications, anatomical reattachment rate, and postoperative best-corrected visual acuity (BCVA).

\section{Results}

There was no statistically significant difference between both groups regarding patients' age, gender, lens status, and the onset of retinal detachment. Seventy percent of both groups presented with inferior retinal detachment while ten percent presented with temporal detachments and twenty percent had a total retinal detachment. Both groups had a statistically significant improvement in postoperative visual acuity in comparison with preoperative visual acuity $(P=0.005$ for group $A$ and $P=<0.001$ for group $B$ ). There was no statistically significant difference between both groups regarding preoperative $(P=0.928)$ and postoperative $(P=0.185)$ visual acuity. A higher incidence of complications was reported in group $A(40 \%)$ in comparison with group $B(30 \%)$ but this difference was not statistically significant $(P=0.69)$. More Epimacular membranes were seen postoperatively in group A (30\%) in comparison with group B $(20 \%)$ but again this difference was not statistically significant $(P=0.657)$. Subretinal hemorrhage was seen in ten percent of cases in both groups. Intraocular pressure was measured in every follow-up of all patients in both groups, no statistically significant difference was found between both groups.

\section{Conclusions}

Both techniques are effective and safe to remove subretinal bands with similar outcomes.

\section{Background}

PVR is a scarring process developing in a portion of retinal detachment cases and is the most common cause of surgical failure of RRD. Growth and contraction of membranes is the hallmark of PVR. 
Contraction may develop on either side of the retina or in the retinal tissue itself.(1) It has been reported that $5-10 \%$ of all retinal detachments develop PVR.(2)

The location of the cellular proliferation will determine the clinical presentation of PVR. For example, if the cellular activity is present on the inner retina, membrane contraction can manifest as a retinal folding or distortion known as a star fold. If cellular proliferation is present in the subretinal space, fibrous strands or irregular plaques are seen.(2)

The risk factors for developing different types of PVR are not well defined. Regression analysis showed a positive correlation of developing subretinal PVR with more retinal quadrants detached, longer duration of $\mathrm{RD}$, atrophic retinal breaks, and younger age.(3)

Pars plana vitrectomy is a technique of choice of many surgeons nowadays for the treatment of RRD. Vitrectomy is necessary for many situations such as in tractional retinal detachments, RRD complicated by PVR, and if the surgical view is hindered by vitreous hemorrhage or dense cataract. The procedure involves the removal of vitreous humor and substituting it with gas or liquid tamponade to seal breaks. During the procedure, breaks are treated by either laser photocoagulation or cryopexy.(4)

Steve Charles developed the punch-through technique for the removal of subretinal bands. A pair of 25gauge retinal forceps is pushed through the retina with the blades closed to access the subretinal space at a point just adjacent to the subretinal band. The blades are then opened to grasp and pull the subretinal band out of the retinotomy created. The endoiluminator can also be used in this technique to act as a fulcrum to increase the tangential pull on the band. The subretinal bands could also be accessed through the original retinal breaks if possible and removed using retinal forceps. Often subretinal bands are dense and fibrous and couldn't be removed safely, in such situations dividing them with scissors through a retinotomy would be enough to relieve the traction.(5)

In 2015 Bernard Wolff developed a new technique for subretinal bands removal through an Ab Externo approach during a scleral buckling operation. The subretinal bands are removed through a 23- or 25gauge valved cannula placed in the subretinal space through the sclera. First, the posterior segment is visualized by an endoilluminator by the help of a non-contact viewing system mounted on an operating microscope. The area of highest detachment is identified and the trocar is advanced at a 15-degree angle to pierce the sclera and choroid at this area. The cannula is then visualized to be placed in the subretinal space and retinal forceps are advanced through it to access the subretinal band. The band is then grasped and pulled out of the eye. A flute needle is then placed in the subretinal space to drain subretinal fluid controllably and the rest of the surgery is completed in the standard fashion. (6)

This novel technique avoids the need for creating a separate retinotomy for subretinal bands removal. A retinotomy may increase the risk of developing epiretinal membranes as shown by a recent study published in the American Journal Of Ophthalmology.(7) While potential complications with the Ab Externo approach include retinal injury and subretinal bleeding.(6) 


\section{Methods}

\section{Patients and study design}

The study was conducted on thirty cases of primary RRD in the Alexandria Main University Hospital. All patients had RRD complicated by subretinal bands interfering with retinal flattening. Patients with recurrent $\mathrm{RD}$, nonrhegmatogenous $\mathrm{RD}$, combined rhegmatogenous and choroidal detachments, and with shallow RD were excluded. Cases were distributed randomly into two groups; ten cases underwent pars plana vitrectomy with subretinal bands removal using the classical Ab-interno approach through one or more retinotomies (group $\mathrm{A}$ ) and twenty cases underwent pars plana vitrectomy with subretinal bands removal through the ab-externo approach.

A detailed history was taken from all patients including patients' age and gender, duration of retinal detachment, and any relevant medical history. The preoperative ocular examination included uncorrected and best-corrected visual acuity, anterior segment examination, and intraocular pressure (IOP) measurement by applanation tonometry. Fundus examination was done with detailed documentation of retinal detachment configuration and location of breaks.

Alexandria University ethics review board approval was obtained and the research followed the tenets of the Declaration of Helsinki. Informed consent was taken from all patients participating in the study after explaining patients' current condition, the surgery needed, benefits, and possible complications of the surgical intervention.

All procedures were done under general anesthesia and informed consent was taken from all patients and any risks involved were explained thoroughly.

\section{Surgical technique:}

Group A: Three valved $23 \mathrm{G}$ cannulas are inserted through pars plana at $3.5 \mathrm{~mm}$ from the limbus in aphakic and pseudophakic patients and $4 \mathrm{~mm}$ from the limbus in phakic patients. One cannula at the inferotemporal position, one at the superotemporal position, and one at the superonasal position. The posterior segment is then visualized using the endoluminator as a light source and wide-field Resight (Carl Zeiss Meditec AG) noncontact viewing system mounted on an operating microscope. Vitrectomy is completed in a standard fashion. Core vitrectomy, posterior vitreous detachment, vitreous base shaving, and identification of breaks. A small retinotomy is created at the highest point of retinal elevation caused by the subretinal band at a point just adjacent to the band. (5) A 23G forceps are introduced through the retinotomy and then opened to grasp, lift, and remove the band. (5) Fluid air exchange, flattening of the retina, and silicone oil injection are then carried out. Pars plana cannulas are then removed and sclerotomies sutured if needed.

Group B: Three valved 23G cannulas are inserted through pars plana at $3.5 \mathrm{~mm}$ from the limbus in aphakic and pseudophakic patients and $4 \mathrm{~mm}$ from the limbus in phakic patients. One cannula at the inferotemporal position, one at the superotemporal position, and one at the superonasal position. 
Infusion line secured at inferotemporal cannula but turned off. A 25G chandelier is insereted at the inferonasal position. Using the endoluminator the cannula is visualized to be passing through the pars plana and not in subretinal space. The posterior segment is then visualized using the endoluminator as a light source and wide-field Resight (Carl Zeiss Meditec AG) non-contact viewing system mounted on an operating microscope. The most elevated point of the retinal detachment is identified and an appropriate external position for access to the subretinal band is chosen. (image 1) During internal visualization, a $23 \mathrm{G}$ trocar is advanced at an angle of around $15^{\circ}$, penetrating the sclera and the choroid, and entering the subretinal space.(image 2) Once it has been visually confirmed that the cannula entered the subretinal space, the trocar is removed and the cannula remains in the subretinal space.(6) Vitreoretinal forceps are then introduced through the valved cannula to grab and remove subretinal membranes through the cannula.(6) (image 3) After complete membrane removal, the cannula can be removed and balanced salt

solution infusion is switched on. ${ }^{(6)}$ Vitrectomy is then completed in a standard fashion. Core vitrectomy, posterior vitreous detachment, vitreous base shaving, identification of breaks, fluid air exchange, flattening of the retina, and silicone oil injection. Pars plana cannulas are then removed and sclerotomies sutured if needed.

Patients in both groups received appropriate postoperative treatment. Patient follow-up was carried at 1day, 1 week, 1 month, and 3 months intervals. The following criteria were assessed: Efficacy in the removal of subretinal bands, complication documentation such as subretinal or vitreous hemorrhage, retinal perforation, and intraoperative hypotony, anatomical success in treating retinal detachment, and functional success as regarding final postoperative visual acuity gain.

\section{Sample size and statistical analysis}

Data were fed to the computer and analyzed using IBM SPSS software package version 20.0. (Armonk, NY: IBM Corp). Qualitative data were described using number and percent. Shapiro-Wilk test was used to verify the normality of distribution. Quantitative data were described using range (minimum and maximum), mean, standard deviation, median and interquartile range (IQR). Significance of the obtained results was judged at the $5 \%$ level. The used tests were, Chi-square test for categorical variables, to compare between different groups, Fisher's Exact or Monte Carlo correction for chi-square when more than $20 \%$ of the cells have expected count less than 5 , Mann Whitney test for abnormally distributed quantitative variables, to compare between two studied groups and Wilcoxon signed ranks test for abnormally distributed quantitative variables, to compare between two periods.

\section{Results}

Table (1) shows the comparison between groups A and B according to age. Regarding patients' ages, group $A$ ranged from thirty-two to sixty-two while group $B$ ranged from twenty-eight to sixty-one. Mean age was $45.33 \pm 8.93$ in group $A$ while was $46.53 \pm 10.29$ in group $B$. there was no significant difference between both groups regarding patients age $(P=0.736)$. 
Group A had seven males (70\%) and three females (30\%) while group B had eleven males (55\%) and nine females (45\%). There was no significant difference between the two groups regarding gender $(P=0.694)$.

Table (2) shows the difference between both groups regarding lens status. Seven patients (70\%) were phakic in group A while only three (30\%) were pseudophakic. On the other hand, twelve patients $(60 \%)$ were phakic in group B and eight (40\%) were pseudophakic. There was no statistically significant difference between both groups regarding lens status $(P=0.702)$.

Table (2) demonstrates the duration of retinal detachment in months in both groups. Both groups had retinal detachment symptoms ranging from two to twenty-four months. Group A had a higher mean of $11.70 \pm 7.24$ months compared to group B's mean of $9.10 \pm 6.23$ months. There was no statistically significant difference between both groups $(P=0.314)$

Inferior retinal detachment was the most common configuration in both groups (70\%) followed by total retinal detachment $(20 \%)$ and then followed by temporal configuration $(10 \%)$.

Table (3) show the comparison between both groups regarding preoperative and final postoperative visual acuities (at 3 months) in decimal. Group B had a slightly better preoperative visual acuity (mean $0.027 \pm 0.030$ in decimal and $-1.98 \pm 0.75$ in logMAR) in comparison with group A (mean $0.026 \pm 0.031$ in decimal and $-1.99 \pm 0.76$ ). On the other hand group $A$ had a better postoperative visual acuity (mean $0.130 \pm 0.063$ in decimal and $-0.94 \pm 0.24$ in logMAR) in comparison with group $B$ (mean $0.101 \pm 0.055$ in decimal and $1.06 \pm 0.25$ in logMAR). There was no statistically significant difference between both groups regarding both preoperative and postoperative visual acuities ( $P=0.928$ and $P=0.185$ respectively).

There was a statistically significant improvement in visual acuity in the postoperative period in comparison with the preoperative period in both groups. P-value was 0.005 for group $A$ and was $<0.001$ for group B.

Table (4) shows the comparison between both groups according to the frequency of complications encountered. A higher frequency of complications was seen with group A $(40 \%)$ in comparison with group $B(30 \%)$. This difference was not statistically significant $(P=0.690)$.

Epimacular membrane formation was a more commonly encountered complication in both groups followed by subretinal hemorrhage. Thirty percent of patients in group A developed epimacular membranes while only ten percent had a subretinal hemorrhage. Twenty percent of patients in group $B$ developed epimacular membranes but only ten percent had a subretinal hemorrhage.

More epiretinal membranes were seen in group A (30\%) in comparison to group B (20\%). This difference was not statistically significant $(P=0.657)$. Ten percent of patients in both groups had subretinal hemorrhage $(P=1.0)$.

Two cases were complicated by subretinal hemorrhage in group B and were encountered intraoperatively during trocar insertion. Fortunately, the hemorrhage was minimal and didn't obscure visualization of the 
subretinal band. The hemorrhage was left to be resolved on its own during the postoperative period.

One case was complicated by a subretinal hemorrhage in group A and was again encountered intraoperatively. The hemorrhage occurred when the end gripping forceps inadvertently touched the choroid while being introduced through the retinotomy to grasp the subretinal band. Immediately the infusion pressure was raised to try and control the bleeding. Fortunately, the hemorrhage was controlled and the minimal amount of blood was aspirated through the retinotomy.

All cases in both groups were silicone-filled at the end of the three months follow-up period. The seven cases in both groups who developed epimacular membranes will be managed by epimacular membrane removal at the time of silicone oil removal.

Table (5) shows the difference between both groups regarding postoperative intraocular pressure at different follow-up periods. Group A had a higher mean postoperative Intraocular pressure at one day, one week, one month, and three months $(17.20 \pm 4.83,14.0 \pm 2.83,14.60 \pm 3.66,14.40 \pm 2.27$ respectively) in comparison with group B mean postoperative intraocular pressure $(17.10 \pm 4.54,13.75 \pm 2.53,14.45 \pm$ $3.75,14.20 \pm 2.14$ respectively), but the difference was not statistically significant $(P=0.956,0.808,0.918$, 0.815 respectively). Patients who had intraocular pressure above 20 in any of the postoperative periods were treated with topical intraocular pressure-lowering medications. All patients with high intraocular pressure responded well to medical treatment and none required extra interventions.

Table (1): Comparison between both groups regarding patients' age.

\begin{tabular}{|lllll|}
\hline & Group A $(\mathbf{n}=\mathbf{1 0})$ & Group $\mathbf{B}(\mathbf{n}=\mathbf{2 0})$ & Test of sig. & $\mathbf{p}$ \\
\hline Age (years) & $32.0-62.0$ & $28.0-61.0$ & & \\
Min. - Max. & $45.33 \pm 8.93$ & $46.53 \pm 10.29$ & & \\
\cline { 1 - 1 } Mean \pm SD. & 43.0 & 47 & $\mathrm{t}=$ & 0.736 \\
\hline Median & & & 0.341 & \\
\hline
\end{tabular}

Table (2): Comparison between both groups regarding gender, lens status, duration of retinal detachment, and retinal detachment configuration. 


\begin{tabular}{|c|c|c|c|c|c|c|}
\hline & \multicolumn{2}{|c|}{ Group $A(n=10)$} & \multicolumn{2}{|c|}{ Group B $(n=20)$} & \multirow{2}{*}{$\begin{array}{l}\text { Test of } \\
\text { sig. }\end{array}$} & \multirow[t]{2}{*}{$p$} \\
\hline & No. & $\%$ & No. & $\%$ & & \\
\hline \multicolumn{7}{|l|}{ Gender } \\
\hline Male & 7 & 70.0 & 11 & 55.0 & \multirow[t]{2}{*}{$x^{2}=0.625$} & \multirow[t]{2}{*}{0.694} \\
\hline Female & 3 & 30.0 & 9 & 45.0 & & \\
\hline \multicolumn{7}{|l|}{ Lens status } \\
\hline Phakic & 7 & 70 & 12 & 60 & \multirow[t]{2}{*}{0.287} & \multirow[t]{2}{*}{0.702} \\
\hline Pseudophakic & 3 & 30 & 8 & 40 & & \\
\hline \multicolumn{7}{|c|}{$\begin{array}{l}\text { Duration of } \\
\text { detachment (months) }\end{array}$} \\
\hline Min. - Max. & $2.0-24.0$ & $2.0-24.0$ & $2.0-24.0$ & $2.0-24.0$ & \multirow{3}{*}{$\begin{array}{l}U= \\
77.50\end{array}$} & \multirow[t]{3}{*}{0.314} \\
\hline Mean \pm SD. & $\begin{array}{l}11.70 \pm \\
7.24\end{array}$ & $\begin{array}{l}9.10 \pm \\
6.23\end{array}$ & $\begin{array}{l}9.10 \pm \\
6.23\end{array}$ & $\begin{array}{l}9.10 \pm \\
6.23\end{array}$ & & \\
\hline Median (IQR) & $\begin{array}{l}11.0(6.0- \\
12.0)\end{array}$ & $\begin{array}{l}9.0(4.5- \\
12.0)\end{array}$ & $\begin{array}{l}9.0(4.5- \\
12.0)\end{array}$ & $\begin{array}{l}9.0(4.5- \\
12.0)\end{array}$ & & \\
\hline \multicolumn{7}{|c|}{$\begin{array}{l}\text { Retinal detachment } \\
\text { configuration }\end{array}$} \\
\hline Inferior & 7 & 70.0 & 14 & 70.0 & & \\
\hline Temporal & 1 & 10.0 & 2 & 10.0 & 0.294 & 1.00 \\
\hline Total & 2 & 20.0 & 4 & 20.0 & & \\
\hline
\end{tabular}

Table (3): Comparison between both groups regarding preoperative and postoperative visual acuity. 


\begin{tabular}{|c|c|c|c|c|c|}
\hline VA in decimal & $\begin{array}{l}\text { Group A } \\
(n=10)\end{array}$ & $\begin{array}{l}\text { Group B } \\
(n=20)\end{array}$ & & $\mathbf{U}$ & p \\
\hline \multicolumn{6}{|l|}{ Preoperative } \\
\hline Min. - Max. & $0.001-0.100$ & $0.001-0.100$ & & \multirow[t]{3}{*}{98.0} & \multirow[t]{3}{*}{0.928} \\
\hline Mean \pm SD & $0.026 \pm 0.031$ & $0.027 \pm 0.030$ & & & \\
\hline Median (IQR) & $0.020(0.001-0.030)$ & $0.020(0.001-0.040)$ & & & \\
\hline \multicolumn{6}{|l|}{ Postoperative } \\
\hline Min. - Max. & $0.050-0.200$ & $0.030-0.200$ & \multirow[t]{3}{*}{71.0} & \multirow{3}{*}{\multicolumn{2}{|c|}{0.185}} \\
\hline Mean \pm SD & $0.130 \pm 0.063$ & $0.101 \pm 0.055$ & & & \\
\hline Median (IQR) & $0.100(0.100-0.200)$ & $0.100(0.050-0.160)$ & & & \\
\hline $\mathbf{p}_{1}$ & $0.005^{*}$ & $<0.001^{*}$ & & & \\
\hline
\end{tabular}

Table (4): Comparison between both groups regarding complications.

\begin{tabular}{|c|c|c|c|c|c|c|}
\hline \multirow[t]{2}{*}{ Complications } & \multicolumn{2}{|c|}{$\begin{array}{l}\text { Group A } \\
(n=10)\end{array}$} & \multicolumn{2}{|c|}{$\begin{array}{l}\text { Group B } \\
(n=20)\end{array}$} & \multirow[t]{2}{*}{$x^{2}$} & \multirow[t]{2}{*}{$\mathrm{FE}_{\mathrm{p}}$} \\
\hline & No. & $\%$ & No. & $\%$ & & \\
\hline Negative & 6 & 60.0 & 14 & 70.0 & \multirow[t]{2}{*}{0.300} & \multirow[t]{2}{*}{0.690} \\
\hline Positive & 4 & 40.0 & 6 & 30.0 & & \\
\hline Epimacular membrane & 3 & 30.0 & 4 & 20.0 & 0.373 & 0.657 \\
\hline Subretinal hemorrhage & 1 & 10.0 & 2 & 10.0 & 0.000 & 1.000 \\
\hline
\end{tabular}

Table (5): Intraocular pressure readings in both groups along follow-up period. 


\begin{tabular}{|c|c|c|c|c|}
\hline Intraocular pressure & $\begin{array}{l}\text { Group A } \\
(n=10)\end{array}$ & $\begin{array}{l}\text { Group B } \\
(n=20)\end{array}$ & $\mathbf{t}$ & $\mathbf{p}$ \\
\hline \multicolumn{5}{|l|}{1 day } \\
\hline Min. - Max. & $14.0-26.0$ & $13.0-27.0$ & \multirow[t]{3}{*}{0.056} & \multirow[t]{3}{*}{0.956} \\
\hline Mean \pm SD. & $17.20 \pm 4.83$ & $17.10 \pm 4.54$ & & \\
\hline Median (IQR) & $15.0(14.0-18.0)$ & $15.0(14.0-18.0)$ & & \\
\hline \multicolumn{5}{|l|}{1 week } \\
\hline Min. - Max. & $10.0-18.0$ & $10.0-18.0$ & \multirow[t]{3}{*}{0.245} & \multirow[t]{3}{*}{0.808} \\
\hline Mean \pm SD. & $14.0 \pm 2.83$ & $13.75 \pm 2.53$ & & \\
\hline Median (IQR) & $13.0(12.0-16.0)$ & $13.0(12.0-16.0)$ & & \\
\hline \multicolumn{5}{|l|}{1 month } \\
\hline Min. - Max. & $12.0-22.0$ & $11.0-23.0$ & \multirow[t]{3}{*}{0.104} & \multirow[t]{3}{*}{0.918} \\
\hline Mean \pm SD. & $14.60 \pm 3.66$ & $14.45 \pm 3.75$ & & \\
\hline Median (IQR) & $13.0(12.0-16.0)$ & $13.50(12.0-15.0)$ & & \\
\hline \multicolumn{5}{|l|}{3 months } \\
\hline Min. - Max. & $12.0-18.0$ & $11.0-18.0$ & \multirow[t]{3}{*}{0.236} & \multirow[t]{3}{*}{0.815} \\
\hline Mean \pm SD. & $14.40 \pm 2.27$ & $14.20 \pm 2.14$ & & \\
\hline Median (IQR) & $14.0(12.0-16.0)$ & $14.0(12.0-15.50)$ & & \\
\hline
\end{tabular}

\section{Discussion}

To our knowledge this is the first study to assess the efficacy and safety of extracting a subretinal band through an Ab-externo approach in a setting of pars plana vitrectomy.

Our study was a randomized prospective study performed on thirty primary rhegmatogenous retinal detachment patients presenting with subretinal bands. Ten patients were assigned to group A where subretinal bands were removed by the classical Ab-interno approach and the remaining twenty patients assigned to group B and subretinal bands removed using the Ab-externo approach. We excluded patients with non-rhegmatogenous retinal detachments, choroidal detachments and patients with shallow retinal detachment.

There was no statistically significant difference between both groups regarding patients age, gender, lens status and onset of retinal detachment. Seventy percent of both groups presented with inferior retinal 
detachment while ten percent presented with temporal detachments and twenty percent had total retinal detachment.

Both groups had a statistically significant improvement in postoperative visual acuity in comparison with preoperative visual acuity ( $\mathrm{P}=0.005$ for group $\mathrm{A}$ and $\mathrm{P}=<0.001$ for group $\mathrm{B})$. There was no statistically significant difference between both groups regarding preoperative $(P=0.928)$ and postoperative $(P=0.185)$ visual acuity.

A higher incidence of complications was reported in group A (40\%) in comparison with group B (30\%) but this difference was not statistically significant $(P=0.69)$. More epimacular membranes were seen postoperatively in group A $(30 \%)$ in comparison with group B $(20 \%)$ but again this difference was not statistically significant $(P=0.657)$. Subretinal haemorrhage was seen in ten percent of cases in both groups. Intraocular pressure was measured in every follow up of all patients in both groups, no statistical significant difference was found between both groups.

\section{Conclusion}

Despite the Ab-externo approach being more technically challenging, we concluded that both techniques are safe and effective in subretinal bands removal in the setting of pars plana vitrectomy. Both techniques had similar outcomes with no statistically significant difference regarding complications. We believe the Ab-externo approach is best for cases with high retinal detachment thus making manipulation in the subretinal space easier and safer. If retinal detachment is shallow, we believe the classical method is a safer option.

\section{Declarations}

Ethics approval and consent to participate: Alexandria University ethics review board approval was obtained and the research followed the tenets of the Declaration of Helsinki. Informed consent was taken from all patients participating in the study after explaining patients' current condition, the surgery needed, benefits, and possible complications of the surgical intervention.

\section{Consent for publication: NA}

Availability of data and materials: datasets generated and analyzed during the current study are not publicly available due to confidentiality issues but are available from the corresponding author on reasonable request.

\section{Competing interests: NA}

Funding: NA

Authors' contributions: SB : Idea of the technique, performing surgeries, reviewed the manuscript 
MG: examining the patients, collecting data, gathering results, writing the draft

AA: reviewed the manuscript

MA: supervising data collection, writing and revising the manuscript.

Acknowledgements: NA

\section{References}

1. Pastor JC, de la Rúa ERg, Martín F. Proliferative vitreoretinopathy: risk factors and pathobiology. Progress in retinal and eye research. 2002;21(1):127-44.

2. Pastor JC. Proliferative vitreoretinopathy: an overview. Survey of ophthalmology. 1998;43(1):3-18.

3. Lewis H, Aaberg TM, Abrams GW, McDonald HR, Williams GA, Mieler WF. Subretinal Membranes in Proliferatlve Vitreoretinopathy. Ophthalmology. 1989;96(9):1403-15.

4. Figueroa MS, Contreras I, Noval S, Group PS. Anatomic and visual outcomes of 23-G vitrectomy without scleral buckling for primary rhegmatogenous retinal detachment. European journal of ophthalmology. 2013;23(3):417-22.

5. Charles S. Vitrectomy techniques for complex retinal detachments. Taiwan Journal of Ophthalmology. 2012;2(3):81-4.

6. Wolff B. Subretinal surgery ab externo: a novel approach to access the Subretinal space without the need for retinotomy. Retina. 2015;35(7):1474-5.

7. Ishikawa K, Akiyama M, Mori K, Nakama T, Notomi S, Nakao S, et al. Drainage retinotomy confers risk of epiretinal membrane formation following vitrectomy for rhegmatogenous retinal detachment repair. American Journal of Ophthalmology. 2021.

\section{Figures}




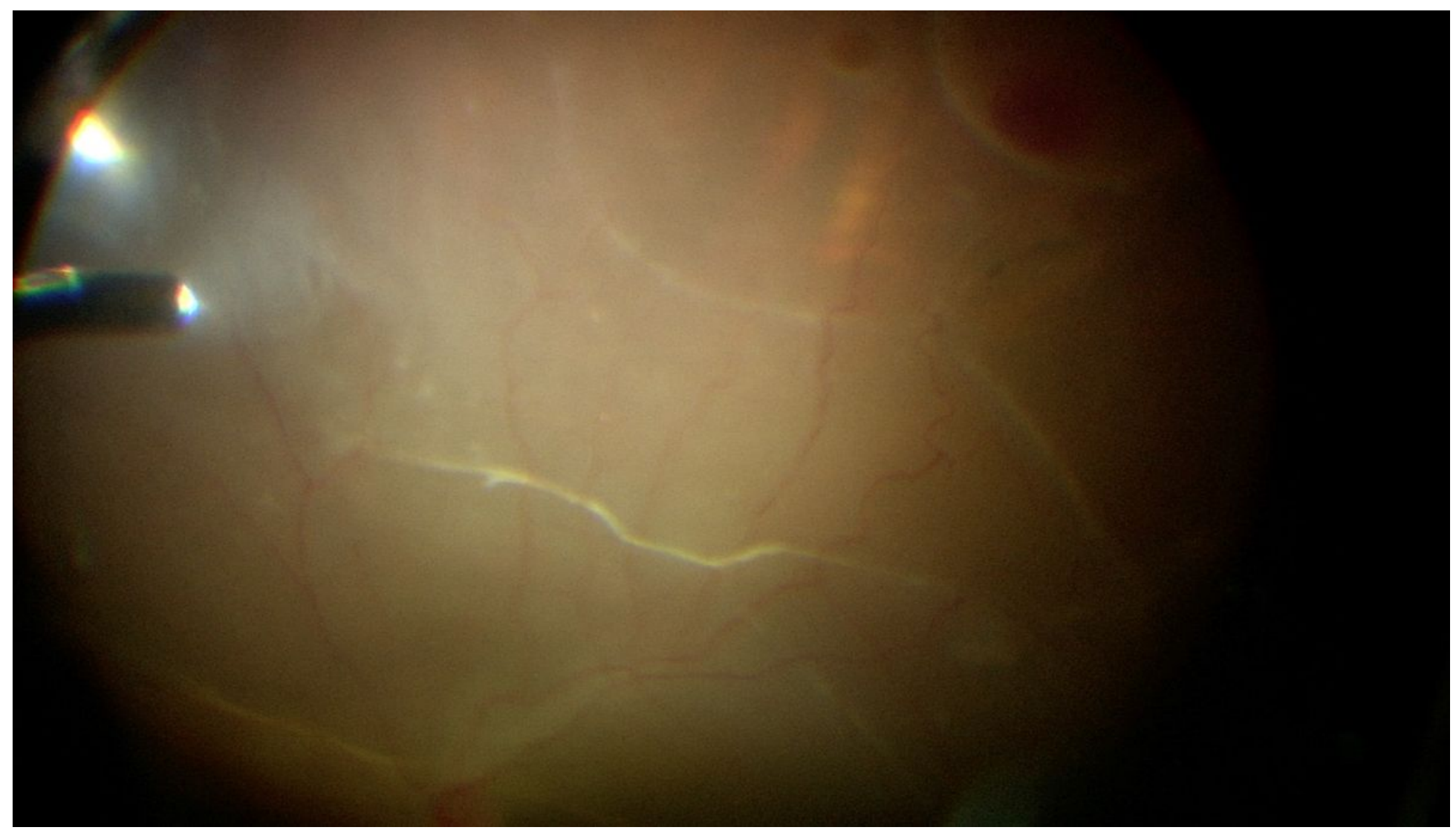

Figure 1

Caption not included with this version.

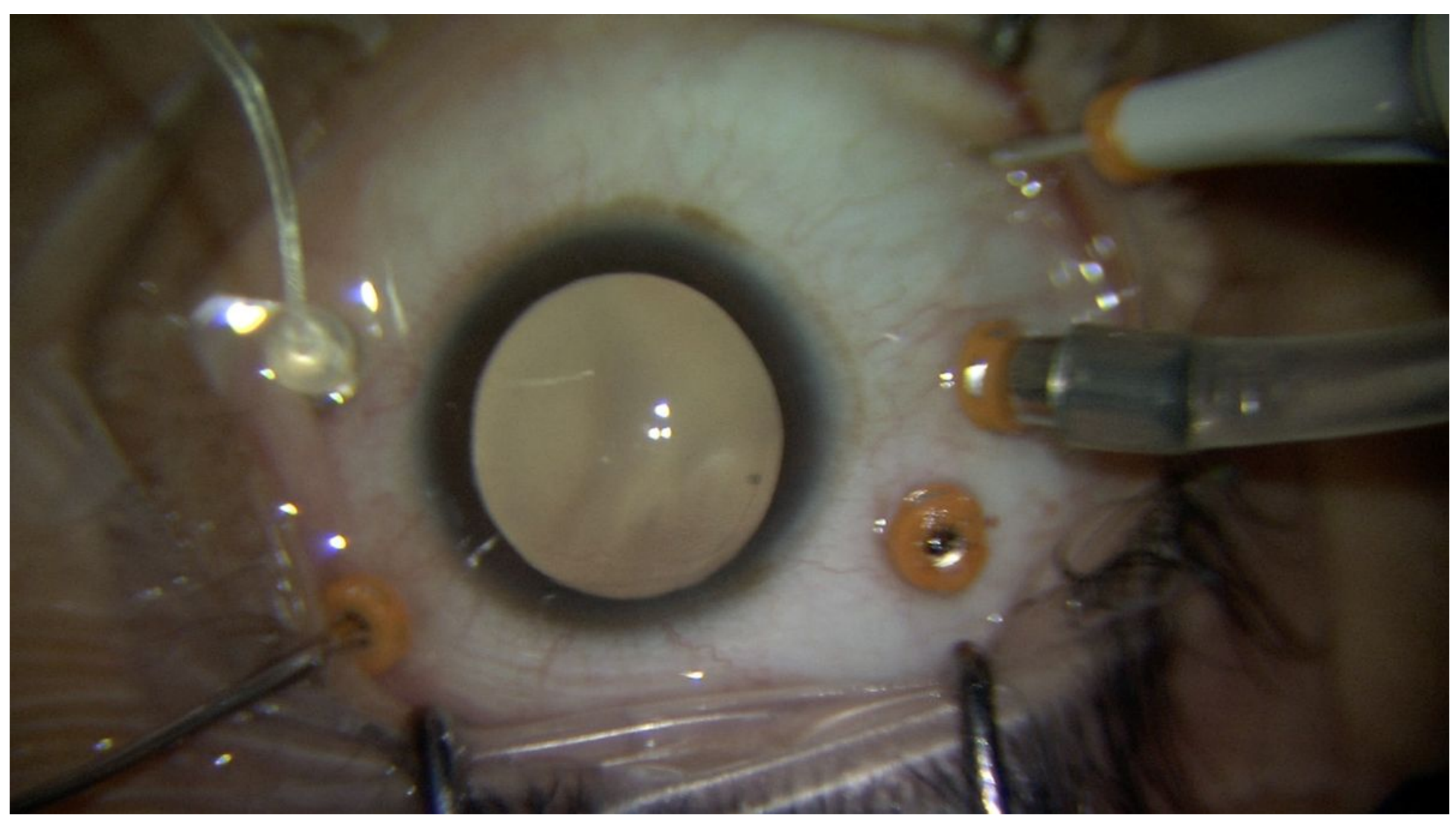

Page 13/14 
Figure 2

Caption not included with this version.

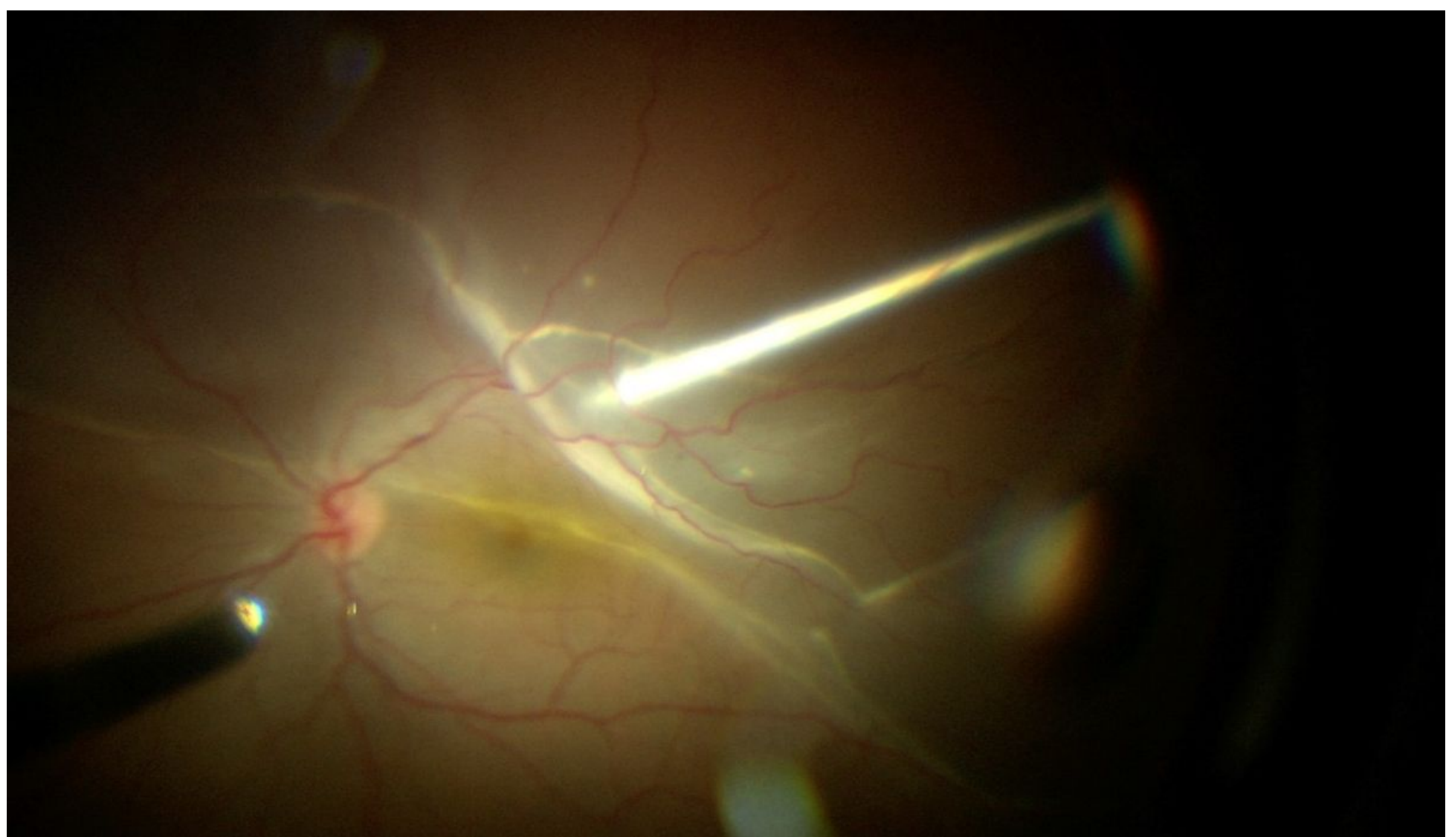

Figure 3

Caption not included with this version.

\section{Supplementary Files}

This is a list of supplementary files associated with this preprint. Click to download.

- GHONEIMx264001.mp4 